\title{
Spherical Harmonics Power-spectrum of Global Geopotential Field of Gaussian-bell Type
}

\author{
Hyeong-Bin Cheong* and Hae-Jin Kong \\ Department of Environmental Atmospheric Sciences, Pukyong National University, Busan 608-737, Korea
}

\begin{abstract}
Spherical harmonics power spectrum of the geopotential field of Gaussian-bell type on the sphere was investigated using integral formula that is associated with Legendre polynomials. The geopotential field of Gaussian-bell type is defined as a function of sine of angular distance from the bell's center in order to guarantee the continuity on the global domain. Since the integral-formula associated with the Legendre polynomials was represented with infinite series of polynomial, an estimation method was developed to make the procedure computationally efficient while preserving the accuracy. The spherical harmonics power spectrum was shown to vary significantly depending on the scale parameter of the Gaussian bell. Due to the accurate procedure of the new method, the power (degree variance) spanning over orders that were far higher than machine roundoff was well explored. When the scale parameter (or width) of the Gaussian bell is large, the spectrum drops sharply with the total wavenumber. On the other hand, in case of small scale parameter the spectrum tends to be flat, showing very slow decaying with the total wavenumber. The accuracy of the new method was compared with theoretical values for various scale parameters. The new method was found advantageous over discrete numerical methods, such as Gaussian quadrature and Fourier method, in that it can produce the power spectrum with accuracy and computational efficiency for all range of total wavenumber. The results of present study help to determine the allowable maximum scale parameter of the geopotential field when a Gaussian-bell type is adopted as a localized function.
\end{abstract}

Keywords: geopotential field, spectral method, Gaussian bell, spherical harmonics, power spectrum

\section{Introduction}

Spatial distribution of scalar variables is often represented with a Gaussian-bell function. It represents a quite natural form of scalar distribution in many physical, geophysical, and statistical quantities. In numerical weather prediction models, the Gaussian bell function is widely used to evaluate the accuracy and stability of the numerical method with which the models are implemented. As is well known, the Gaussian bell shows a symmetric structure and it decays exponentially away from the bell's center (Hopkins, 1973; Cheong and Park, 2008; Cheong et

\footnotetext{
*Corresponding author: hbcheong@pknu.ac.kr Tel: +82-51-629-6642

Fax: +82-51-629-6636
}

This is an Open-Access article distributed under the terms of the Creative Commons Attribution Non-Commercial License (http:// creativecommons.org/licenses/by-nc/3.0) which permits unrestricted non-commercial use, distribution, and reproduction in any medium, provided the original work is properly cited. al., 2012). The spatial gradient is zero over the center, and decreases as the distance from the center increases until it reaches a minimum value. Beyond the point of minimum gradient, the slope again increases, and reaches asymptotically zero far away from the center. It should be reminded, however, that the slope goes to zero only when the distance from the center is infinite. Therefore, when a vanishing gradient is required at boundaries of domain of interest, the Gaussian function cannot be used without modification.

Gaussian bell is characterized by one single parameter which specifies the scale. When the term 'width' for the Gaussian bell is used, it means a distance from the center at which the function is decreased by the factor of $e$, which is called as $e$ folding distance hereafter. For this reason, the $e$ folding distance is often used as a scale of the Gaussian bell. If a Gaussian bell of a certain scale is decomposed into sinusoidal functions, i.e., Fourier transformed, the spectrum (or coefficients) of Fourier components will be wide-spread rather than being 
localized around a particular wavenumber. Distribution of spectrum is known to be dependent on the scale parameter. In discrete models the spectrum distribution has very important implication because two broad spectrum may be a cause of aliasing in the discrete models. Therefore, when a Gaussian bell function is used in discrete models, a priori knowledge of the spectrum would help to determine the appropriate resolution of the discrete model. The spectrum of a Gaussian bell is easily obtained by usual Fourier transform for planar coordinates such as Cartesian coordinate system. However, it is not trivial in the case of spherical coordinate systems since Fourier analysis and synthesis are not applicable. Of course, the power spectrum can be obtained by numerical methods such as the Legendre spectral method. In this case, however, the spectrum suffers from the machine round-off, not being able to represent the power spectrum below the machine round off in spite that real power spectrum spans from order one to order to several hundreds. So it is necessary to develop a direct method to estimate the power spectrum of the Gaussian bell with rigorous theoretical approach.

In this study the spherical harmonics spectrum of Gaussian bell function, which is continuous on the global domain, is evaluated using an accurate estimation method based on the integral formula of Legendre polynomials. The Legendre functions and their Fourier-series representation for the purpose of spherical-harmonics spectrum analysis are described in the following section. In Section 3, details of the direct method, two spectral method to calculate the spectrum are presented along with the evaluation result of the accuracy associated with the methods. Conclusions are given in the final section.

\section{Legendre functions and Fourier-series representation}

The Legendre polynomials are the eigenfunctions of the spherical Laplacian operator of zonal wavenumber zero (Dilts, 1985; Enomoto et al., 2004; Cheong et al., 2012):

$$
\frac{d}{d x}\left(1-x^{2}\right) \frac{d}{d x} P_{n}(x)+[n(n+1)] P_{n}(x)=0
$$

where $x=\cos \theta$ with $\theta$ meaning the colatitude, $m$ is the zonal wavenumber, $P_{n}(x)$ represents the Legendre function with order $m$ and degree $n$. In terms of the Rodrigues' formula, the Legendre polynomial $P_{n}(x)$ can be expressed by

$$
P_{n}(x)=\frac{1}{2 ! n !} \frac{d^{n}}{d x^{n}}\left(1-x^{2}\right)^{n}
$$

On the whole domain on the spherical surface, the Legendre polynomials constitute the orthonormal basis such that

$$
\int_{-1}^{+1} P_{n}(x) P_{l}(x) d x=\delta_{n, l}
$$

where $\delta_{n, l}$ means Kronecker delta. From (2), it is easy to represent the Legendre functions of $m=1$ in terms of differentiation of Legendre polynomials:

$$
P_{n}^{1}(x)=\sqrt{\frac{1-x^{2}}{n(n+1)}} \frac{d}{d x} P_{n}^{0}=-\sqrt{\frac{1}{n(n+1)}} \frac{d}{d \theta} P_{n}^{0}
$$

As was shown in Cheong et al. (2012), the Legendre functions $P_{n}^{m}(x)$ are represented with polynomials of $x$ (polynomials with the metric factor of $\sqrt{1-x^{2}}$, respectively) for $m=0,2,4, \ldots$ (for $m=1$, $3, \quad 5, \ldots$, respectively). Therefore the Legendre functions can be written in terms of Fourier series as (Hofsommer and Potters, 1960; Dilts, 1985; Moriguchi et al., 1990; Cheong, 2000; Jekeli et at., 2007; Cheong et al., 2012):

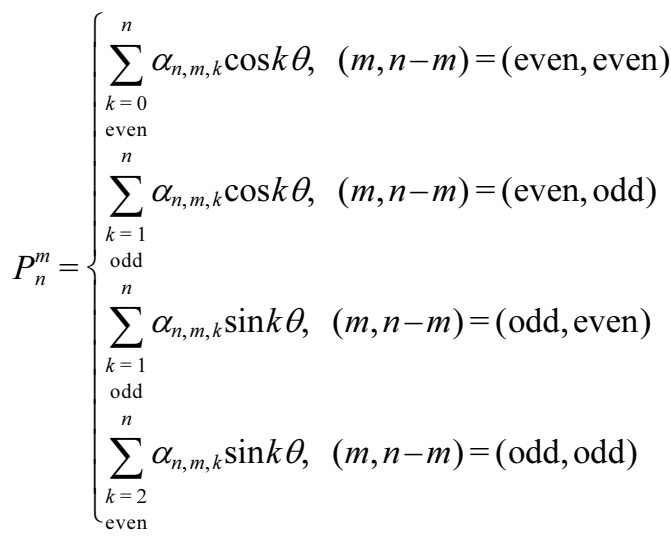


where $\alpha_{n, m, k}$ denotes the Fourier-series coefficient of the Legendre function $P_{n}^{m}(x)$. The number of Fourier components should be the same as the degree. This implies that the trigonometric function $\cos n \theta$ is expressed as a linear combination of Legendre functions of even $m$, while the functions $\sin n \theta$ is expressed with those of odd $m$. For example, only limited number of Legendre function up to $n$ is included in the combination to express the trigonometric functions in case of $m=0$ and 1 (Risbo, 1996; Ricardi and Burrows, 1972; Cheong et al., 2012):

$$
\begin{aligned}
\cos 2 n \theta & =\sum_{k=0}^{n} \alpha_{0,2 k} P_{2 k}^{0} \\
\cos (2 n+1) \theta & =\sum_{k=0}^{n} \alpha_{0,2 k+1} P_{2 k+1}^{0} \\
\sin (2 n+1) \theta & =\sum_{k=0}^{n} \alpha_{1,2 k+1} P_{2 k+1}^{1} \\
\sin 2 n \theta & =\sum_{k=1}^{n} \alpha_{1,2 k} P_{2 k}^{1}
\end{aligned}
$$

where $\alpha_{m, k}$ denotes the Fourier-series coefficient of Legendre functions of $m=1$ and $m=1$. The Fourierseries coefficients can be obtained by the integral formula of the Legendre functions as was presented in Cheong et al. (2012).

\section{Spherical harmonics spectrum of geopotential field of Gaussian bell function}

\section{Direct method based on the Legendre polynomial}

Since the spherical harmonics are isotropic on the global domain, the spherical harmonics spectrum of a Gaussian bell should remain the same regardless of the location on the spherical surface (Sardeshmukh and Hoskins, 1984). Therefore, in this study a Gaussian bell which is centered at the North pole is considered for simplicity:

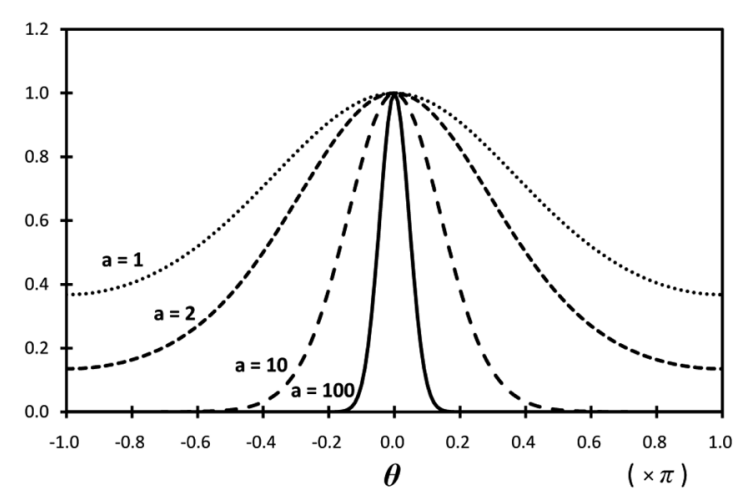

Fig. 1. Cross section of Gaussian bell for different scale parameter $a$, where $\theta$ means the arc-distance from the bell center along great circle.

$$
y(\theta)=\exp \left[-a \sin ^{2}(\theta / 2)\right]
$$

where $\theta$ is the colatitude as in (1), and $\alpha[\geq 1]$ is a scale parameter, with a larger (smaller) value defining a narrow (wide) bell. The function in (7) is continuous on the spherical surface because $y(\pi)=y(-\pi)$ along with $y^{\prime}=0$ at $\theta=\pi$. Some examples of the Gaussian bell, given by the cross section along the great circle passing the center, are illustrated in Fig. 1. [see also Fig. 4(b) for the distribution over the whole sphere where the rotated coordinates are used for better visualization] Integration of $y^{2}$ over the globe can be obtained analytically, and hence will be useful in assessing the estimation method to get the Legendre polynomial coefficient:

$$
\begin{aligned}
\langle y\rangle & \equiv \int_{0}^{\pi} \int_{0}^{2 \pi} y^{2} \sin \theta d \lambda d \theta \\
& =2 \pi \int_{0}^{\pi} \exp \left[-2 a \sin ^{2}(\theta / 2)\right] \sin \theta d \theta \\
& =\frac{2 \pi}{a}\left(1-e^{-2 a}\right)
\end{aligned}
$$

Equation (7) can be represented as a series of $\cos \theta[\equiv x]$, and equivalently a series of the Legendre polynomials:

$$
\begin{aligned}
y(\theta) & =\sum_{k=0}^{\infty} c_{k} \cos _{k} \theta \\
& =\sum_{n=0}^{\infty} \omega_{n} P_{n}(\cos \theta)
\end{aligned}
$$


where the Legendre polynomial is expressed by the Rodrigues' formula in (2), and is orthonormal as in (3).

The power series coefficient in (9) is obtained through successive differentiation with respect to colatitude and applying the pole conditions as in Cheong and Park (2007, 2008), and the Legendre coefficients can be obtained by projecting the Legendre polynomials to the function in (7):

$$
\begin{aligned}
c_{k} & =\frac{1}{k !}\left(-\frac{a}{2}\right)^{k} \exp \left(-\frac{a}{2}\right) \\
\omega_{n} & =\int_{-1}^{+1} y(\theta) P_{n}(x) d x \\
& =\int_{-1}^{+1}\left[\sum_{k=0}^{\infty} c_{k} x^{k}\right] P_{n}(x) d x \\
& =\sum_{k=0}^{\infty}\left[c_{k} \int_{-1}^{+1} x^{k} P_{n}(x) d x\right] \\
& =\sum_{r=0}^{\infty}\left[c_{2 r+n} \int_{-1}^{+1} x^{2 r+n} P_{n}(x) d x\right]
\end{aligned}
$$

where $\int_{-1}^{1} x^{k} P_{n}(x) d x=0 \quad$ for $\quad k<n$ was used. Substituting (10) into (9), the integral in the last line of (11) is represented as

$$
\begin{aligned}
Z_{n, r} & =\int_{-1}^{+1} c_{2 r+n} x^{2 r+n} P_{n}(x) d x \\
& =e^{-a / 2}(-a / 2)^{2 r+n} \frac{2^{n} \sqrt{2(2 n+1)}(r+n) !}{(r !)(2 r+2 n+1) !}
\end{aligned}
$$

where the integral formula of Legendre functions was incorporated (Moriguchi et al., 1990):

$$
\int_{-1}^{+1} x^{2 r+n} P_{n}(x) d x=2^{n} \sqrt{2(2 n+1)} \frac{(2 r+n) !(r+n) !}{(r !)(2 r+2 n+1) !}
$$

The series in (11) include infinite terms with the index $r$, of which magnitude (i.e., absolute value of $Z_{n, r}$ ) may vary from the order $O(1)$ or higher to extremely low order far beyond the machine limit of exponent, e.g., $O\left(10^{-323}\right)$ in the case of double precision processing for FORTRAN90. To make the computation practical, it is necessary to restrict the number of terms to a finite value by examining relative importance of the terms. Recalling the fact that $Z_{n, r} \rightarrow 0$ as $r \rightarrow \infty$, the choice of terms of relative importance can be done by considering two possible behaviors of $Z_{n, r}$ : One is the case where it decreases monotonously with $r$, and hence the summation should start from $r=0$. The other is the case where it has a local maximum at which $Z_{n, r}$ decreases monotonously, and therefore the summation starts from it and is proceeded to both sides. The summation continues until the next term is smaller than the sum by the order of a predetermined value, being in this case $O\left(10^{-17}\right)$, which is very close to the machine rounding error in the double-precision computations.

Search of the local maximum point of $Z_{n, r}$ is done by taking the ratio of two adjacent terms, and demanding that it approaches to unity:

$$
\frac{Z_{n, r+1}}{Z_{n, r}}=\frac{1}{2}\left(\frac{a}{2}\right)^{2} \frac{1}{(r+1)(2 r+2 n+3)}=1
$$

which yields, from the condition of $r>0$, the following relationship

$$
\left\{\begin{array}{l}
n<\frac{1}{4}\left(\frac{a}{2}\right)^{2}-\frac{3}{2}: Z_{n, r} \text { has a local maximum } \\
n \geq \frac{1}{4}\left(\frac{a}{2}\right)^{2}-\frac{3}{2}: Z_{n, r} \text { monotonously decreasing with } r
\end{array}\right.
$$

and

$$
r_{m}=\operatorname{int}\left\{\frac{-(2 n+5)+\sqrt{(2 n+5)^{2}-8\left(2 n+3-a^{2} / 8\right)}}{4}\right\}
$$

In the summation of (11), to avoid a heavy computation associated with factorials, a direct evaluation of $Z_{n, r}$ is done only once and is proceeded in the remaining steps with the use of (14), that is,

$$
Z_{n, r+1}=\left[\frac{1}{2}\left(\frac{a}{2}\right)^{2} \frac{1}{(r+1)(2 r+2 n+3)}\right] Z_{n, r}
$$

In Table 1 , some examples of $r_{m}$ and $n_{c}$, being 
Table 1. The critical wavenumber $n_{c}$ above which the function $Z_{n, r}$ of (17) has a local maximum for a given scale parameter $a$, and the point of local maximum $r_{m}$ for selected values of wavenumber $n$

\begin{tabular}{cccccc}
\hline \hline$a$ & 1 & 10 & 100 & 1000 & 10000 \\
\hline$n_{c}$ & 0 & 5 & 624 & 62449 & 6249999 \\
$\left(n, r_{m}\right)$ & $r_{m}=0$ for all $n$ & $r_{m}=0$ for all $n$ & $(2,22)(50,9)(100,4)$ & $(2,247)(50,225)(100,203)(2,2497)(50,2473)(100,2449)$ \\
\hline
\end{tabular}

Table 2. Spherical harmonics coefficients for $(n, m)=(100,0)$ of Gaussian bell, estimated by the Legendre-polynomial integration formula for scale parameters of $1,10,100,1000$, and 10000

\begin{tabular}{cc}
\hline \hline$a$ & $w_{n=100}$ \\
\hline 1 & $0.716394370983061 \mathrm{E}-218$ \\
10 & $0.845850906458664 \mathrm{E}-120$ \\
100 & $0.905641086590325 \mathrm{E}-037$ \\
1000 & $0.843377454763924 \mathrm{E}-006$ \\
10000 & $0.730207857341843 \mathrm{E}-003$ \\
\hline
\end{tabular}

integers satisfying (15) and (16), are illustrated for various scale parameter. Note that the local maximum does not appear at smaller scale parameter but begins to appear at $a=10$. The critical wavenumber $n_{c}$ increases with the scale parameter, which means that the function $Z_{n, r}$ tends to have the local maximum unless the wavenumber $n$ is quite large, as can be seen in Table 1. The Legendre-polynomial coefficient of the Gaussian bell for $n=100$, obtained through the procedures explained above, is presented in Table 2 for the same scale parameters as in Table 1. The coefficient becomes large as the scale parameter increases, implying flattened distribution of coefficients for large scale parameter (i.e., narrow bell).

From the orthogonality of Legendre polynomials, the global integration of the Gaussian bell squared in (8) is written in terms of Legendre-polynomial coefficients:

$$
\begin{aligned}
\left\langle y^{2}\right\rangle & \equiv \int_{0}^{\pi} \int_{0}^{\pi} y^{2} \sin \theta d \lambda d \theta \\
& =2 \pi \int_{0}^{\pi}\left[\sum_{n=0}^{\infty} \omega_{n} P_{n}(\cos \theta)\right]^{2} \sin \theta d \theta \\
& =2 \pi \sum_{n=0}^{\infty} \omega_{n}^{2}
\end{aligned}
$$

which can be used to check the validity of the estimation method by taking the difference from the theoretical one in (8).

\section{Spectral method with Gaussian quadrature and Fourier-series method}

For the Gaussian bell whose center is given as $\left(\lambda_{c}\right.$, $\theta_{c}$ ), the spherical harmonics spectrum of the Gaussian bell can be obtained by expanding with a finite series of spherical harmonics (Nehrkorn, 1990; Swarztrauber, 1993):

$$
\begin{aligned}
y(\lambda, \theta) & =\operatorname{Re}\left[\sum_{n=|m| n=-M}^{M} \sum_{n}^{M} \hat{y}_{n, m} Y_{n}^{m}(\lambda, \cos \theta)\right] \\
Y_{n}^{m} & =P_{n}^{|m|}[\cos m \lambda+i \sin m \lambda]
\end{aligned}
$$

where $\operatorname{Re}[\cdot]$ implies real part of $\bullet, i=\sqrt{-1}, M$ is the largest total wavenumber, and $\hat{y}_{n, m}$ is the spectral coefficient. It follows $\hat{y}_{n, m}=\hat{y}_{n,-m}^{*}$ from the fact that the function $y(\lambda, \theta)$ is real-valued, and $Y_{n}^{m}=Y_{n}^{m^{*}}$, where the superscript * represents the complex conjugate. The meridional function $P_{n}^{m}$ is the normalized associated Legendre function of order $m$ and degree $n$, which is defined as

$$
P_{n}^{m}(x)=\sqrt{\frac{2 n+1}{2}} \sqrt{\frac{(n-m) !}{(n+m) !}}\left(1-x^{2}\right)^{m / 2} \frac{d^{m}}{d x^{m}} P_{n}(x)
$$

The spectral coefficient is obtained through projection of the spherical harmonics using orthogonality as

$$
\begin{aligned}
\hat{y}_{n, m} & =\int_{0}^{\pi} \frac{1}{2 \pi} \int_{0}^{2 \pi} y(\lambda, \theta) Y_{n}^{m *} \sin \theta d \lambda d \theta \\
& =\int_{0}^{\pi} y_{m} P_{n}^{|m|}(\cos \theta) \sin \theta d \theta \\
y_{m} & =\frac{1}{2 \pi} \int_{0}^{2 \pi} y(\lambda, \theta) e^{-i m \lambda} d \lambda
\end{aligned}
$$




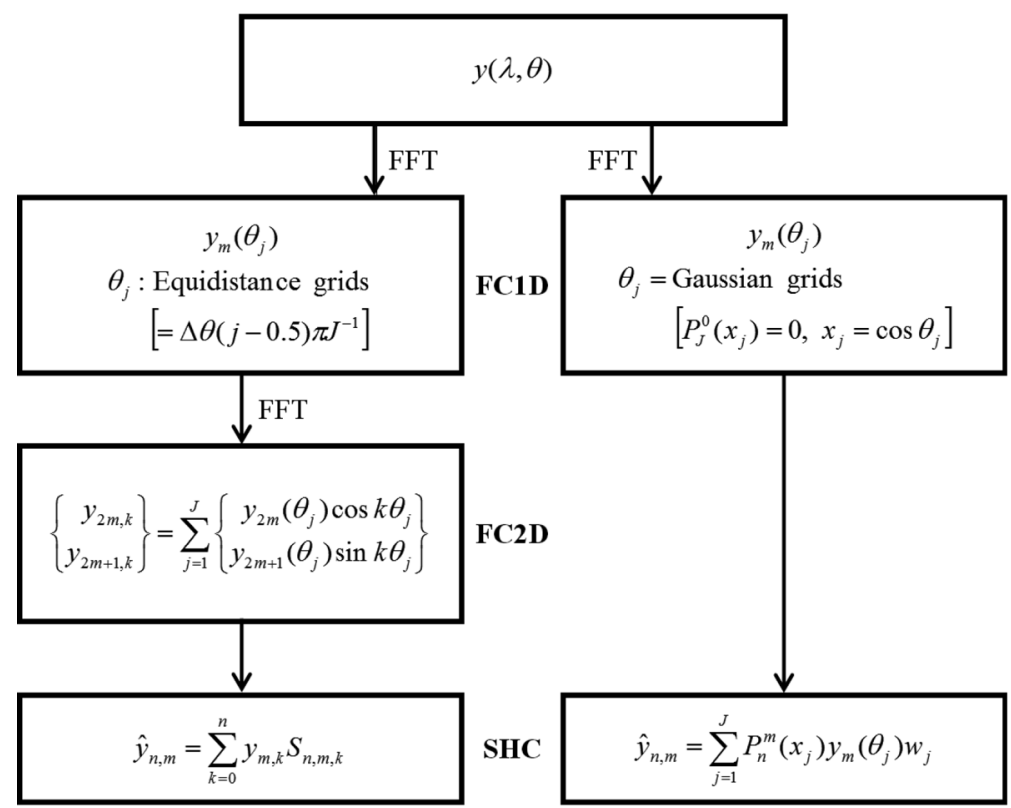

Fourier method

\section{Gaussian quadrature}

Fig. 2. Flowchart for projection of Legendre functions by Fourier method and Gaussian quadrature. FC1D $\left(y_{m}\right)$, FC2D $\left(y_{m, k}\right)$, and SHC represent Fourier coefficients of one-dimension, two-dimension, and spherical harmonics coefficient $\left(\hat{y}_{n, m}\right)$, respectively. The operation count for FC1D and FC2D is only $O\left(J^{2} \log _{2} J\right)$ due to availability of Fast Fourier Transform (FFT), while it is $O\left(J^{3}\right)$ for SHC, where $J$ is the number of Gaussian grid (with the Gaussian weights $\omega_{j}$ ) in the zonal direction.

The spherical harmonics spectrum of the Gaussian bell, given as grid point values on the spherical domain, can be obtained either by the Fourier-series method or the Gaussian quadrature (Sneeuw and Bun, 1996; Rod Blais, 2008; Wittwer et al., 2008; Cheong et al., 2012). The projection of Legendre functions is performed by the integral formula on the global domain for the Fourier-series method, while it is done by weighted sum of gridpoint values on Gaussian grids for the Gaussian quadrature method (Swarztrauber, 1993; Cheong et al., 2012). Two discrete methods, explained above, for the computation of spherical harmonics coefficients are compared in Fig. 2 with a simplified flowchart. Computational efficiency is almost the same for the two methods, but a slightly increased operation count for the Fourier method due to the procedure to calculate two-dimension Fourier coefficients, denoted as FC2D (two dimensional Fourier coefficients) in the figure. The additional computation needed for Fourier method is insignificant because the heaviest burden, represented with $O\left(\hat{J}^{\hat{\beta}}\right)$, is laid on the step to get spherical Harmonics coefficients rather than FC2D which needs only $O\left(J^{2} \log _{2} J\right)$ for $2 J \times J$ grids.

The power spectrum $\left(\omega_{n}\right)$ of the Gaussian bell in terms of total wavenumber (i.e., the degree $n$ ) is obtained by adding the spectral components for all zonal wavenumber:

$$
\omega_{n}=\sqrt{2 \pi \sum_{m=0}^{n} \hat{y}_{n, m} \hat{y}_{n, m}^{*}}
$$

The magnitude of coefficients of $\omega_{n}$ as a function of $n$ is provided in Fig. 3, along with those calculated by two discrete methods for selected scale parameters. It is noted that the spectrum decreases monotonically with the total wavenumber, showing nuch flat structure for increased scale parameter $a$. Two spectral methods do not exhibit significant difference for all scale parameters shown here. It appears that the spectrum obtained by spectral methods do not represent the 

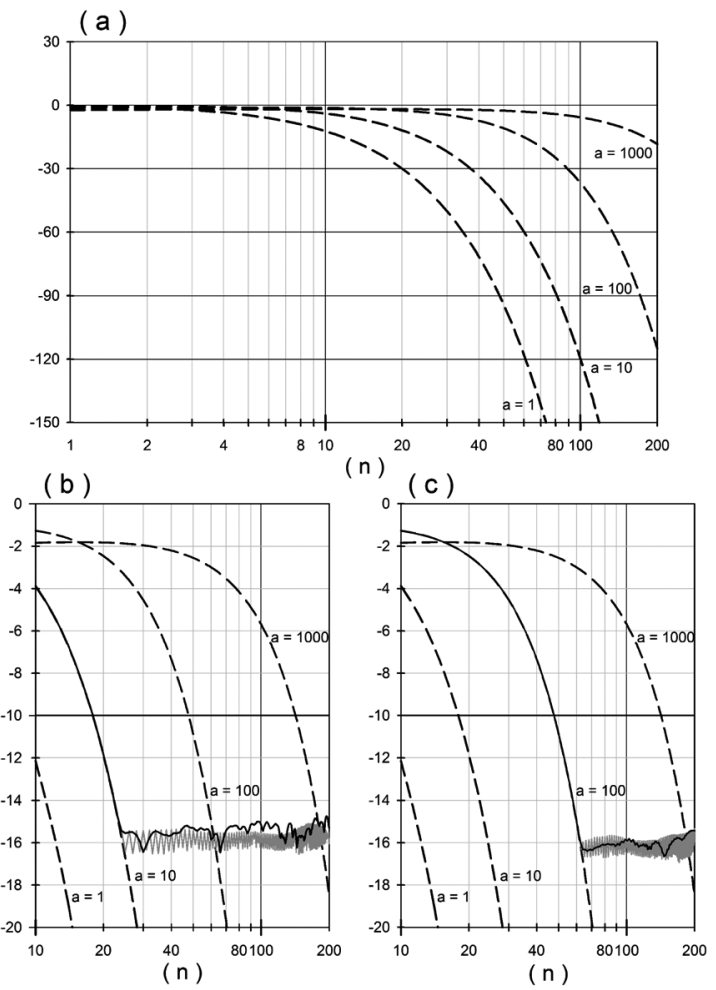

Fig. 3. Logarithmic spherical-harmonics power spectrum of Gaussian bell (i.e., $\log _{10}\left|\omega_{n}\right|$ ) with log-scale for the spherical total-wavenumber (or degree) $n$ on the abscissa. (a): Power spectrum calculated by the estimation method for four scale parameters of $a=1,10,100$, and 1000. (b): Power spectrum for $a=10$ calculated by discrete methods of Gaussian quadrature (thick solid line in black) and Fourier method (thin solid line), where the dashed curves are those in panel (a). (c): Same as (b) but $a=100$. Green lines for small wavenumbers are invisible in (b) and (c) due to complete overlapping with the back solid line.

behavior at larger total wavenumbers. For example, the spectrum remains in the order of machine roundoff beyond $n=23(n=65)$ in the case of $a=10 \quad(a=100)$ due to the truncation error of digital computing with double precision. These provide important implication that the resolution of the discrete model which incorporates the Gaussian-bell function for geopotential field or others should be determined with care in such a way that the information of the Gaussian field is fully taken into consideration: In case of $a=100$, the largest wavenumber of the model should be given greater than $n=65$.
Table 3. Normalized errors of three methods to compute global integration of Gaussian-bell squared for two scale parameters of 10 and 100

\begin{tabular}{cccc}
\hline \hline \multirow{2}{*}{$a$} & \multirow{2}{*}{$\begin{array}{c}\text { Estimation with } \\
\text { integration } \\
\text { formula }\end{array}$} & $\begin{array}{c}\text { Fourier } \\
\text { method }\end{array}$ & $\begin{array}{c}\text { Gaussian } \\
\text { quadrature }\end{array}$ \\
\cline { 3 - 4 } & $0.2322 \mathrm{E}-15$ & $0.1767 \mathrm{E}-15$ & $0.1767 \mathrm{E}-14$ \\
10 & $0.6753 \mathrm{E}-16$ & $0.2209 \mathrm{E}-15$ & $0.6626 \mathrm{E}-15$ \\
\hline
\end{tabular}

\section{Evaluation of the accuracy}

The accuracy of the spectrum of the Gaussian bell can be checked by taking global integration of Gaussian-bell squared and comparing it with the theoretical values. The error normalized by the reference solution in (8) is given as

$$
\frac{\sum_{n=0}^{\infty} \omega_{n}^{2}-a^{-1}\left(1-e^{-2 a}\right)}{a^{-1}\left(1-e^{-2 a}\right)}
$$

Eq. (23) was calculated for the three methods, the estimation by integration formula and two discrete methods of Fourier method and Gaussian quadrature (Table 3). As can be seen in Table 3, three methods present comparable performance showing the errors near machine rounding. The Fourier method produced better results than the Gaussian quadrature, but slightly poor results than the estimation method as a whole. This results indicate that the direct method is the most reliable way to calculate the spherical-harmonics power spectrum of geopotential or any scalar variables of Gaussian-bell type.

The spectral coefficients, as obtained in section 3.2, can be transformed to the gridpoint values by reversing the processes associated with the spectral analysis. One of the advantages of the two spectral methods, found not in the direct method, is that the spectral components can be manipulated with ease. For instance, elimination of some parts of the spectral coefficients, being usually smaller horizontal scales (i.e., larger $n$ ), will result in isotropic filtering (or smoothing) of the given data (Hopkins, 1973; Sardeshmukh and Hoskins, 1984; Enomoto et al., 2004; Jekili et al., 2007; Cheong et al., 2012): 

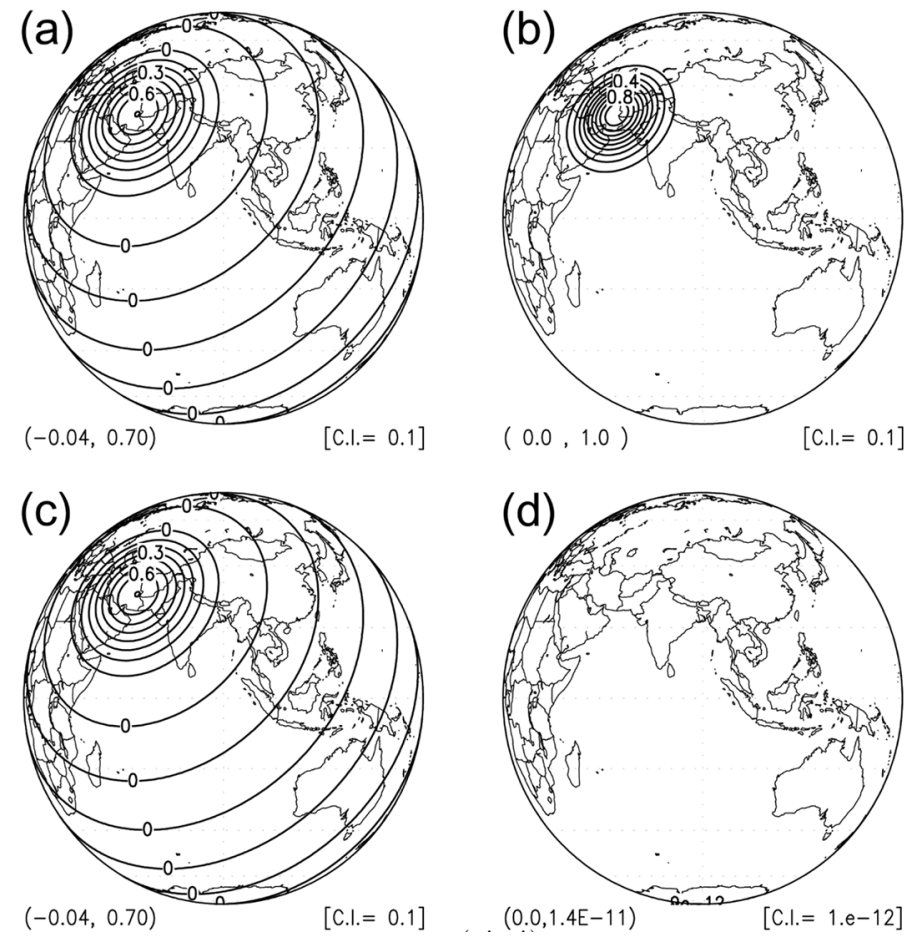

Fig. 4. Panel (a): Filtering of Gaussian bell function using Fourier method, where the small scales with $n>10$ were removed when reconstructing the grid point data from the spherical harmonics coefficients. (b): Original Gaussian bell given by $y(\beta)=\exp \left[-100 \sin ^{2}(\beta / 2)\right]$, where $\beta$ is the angular distance measured from the rotated coordinates $\left(\lambda^{\prime}, \theta^{\prime}\right)$ whose north pole is located at $\left(60^{\circ} \mathrm{E}, 30^{\circ} \mathrm{N}\right)$. (c): Same as (a) except Gaussian quadrature. (d): difference between (b) and (c). The minimum value, the maximum, and contour interval $(\mathrm{CI})$ of the Gaussian bell function are presented in the lower part of each map.

$$
y_{f}(\lambda, \theta)=\operatorname{Re}\left[\sum_{n=|m| n=-M_{f}}^{M_{f}} \sum_{n}^{M_{f}} \hat{y}_{n, m} Y_{n}^{m}(\lambda, \cos \theta)\right]
$$

where $y_{f}$ means filtered field and $M_{f}$ the largest scale retained in the filtered field. In Fig. 4, one example of the filtering is illustrated in the case of $a=10$, where the scales with $n>10$ were removed at the time of reconstruction of gridpoint values from spherical harmonics coefficients. Two methods seem to have produced almost identical results, with the maximum difference of gridpoint-value as small as $O\left(10^{-11}\right)$. It is obvious that as the scale parameter of the Gaussian bell becomes large, the wavenumber truncation $M$ (and also the number of Gaussian grids $J$ ) should be large enough to be away from aliasing error. As far as the Gaussian bell is concerned, the proper choice of $M$ (and also $J$ ) can be determined in the discrete methods by investigating the power spectrum in the reference solution. Considering that the leading order of the Gaussian bell is close to $O(1)$ and the power spectrum is only significant up to the values $O\left(10^{-15}\right)$ $O\left(10^{-16}\right)$ for double precision computations, the number $M$ should be larger than the scale $n$ at which the power begins to drop below.

\section{Conclusion}

The spherical harmonics power spectrum of geopotential of Gaussian-bell function was calculated based on the direct method, the Legendre spectral methods, and Fourier-series spectral method. The Gaussian-bell used in this study is a continuous function on the whole sphere without the discontinuity, unlike a usual Gaussian-bell function which is discontinuous over the opposite point of the bell 
center. Therefore the Gaussian-bell function defined here is appropriate for the evaluation of spherical harmonics power spectrum. Over a wide range of scale parameter of the Gaussian bell, it was found that three methods produced almost the same accuracy, but the direct method was shown to be more accurate than any of the two spectral methods. The most important advantage of the direct method, which is based on the power series of the Legendre polynomials, is that the spectrum can be estimated in the range of infinite order, far beyond the machine round-off inherent in the spectral methods. In other words, since the results of the Fourier method and Legendre spectral method are subject to the digits of accuracy, like other discrete method such as Gaussian quadrature, increased digits of accuracy is required when calculating correctly the power spectra of spherical-surface function or discrete data over extended range, as was demonstrated vividly by Fig. 3 .

\section{Acknowledgments}

This work was supported by a Research Grant of Pukyong National University (year 2013). The authors would like to appreciate helpful comments from anonymous reviewers.

\section{References}

Cheong, H.B., Double Fourier Series on a Sphere, 2000, Applications to Elliptic and Vorticity Equations. Journal of Computational Physics, 157, 327-349.

Cheong, H.B. and Park J.R., 2007, Geopotential field in nonlinear balance with the sectoral mode of RossbyHaurwitz wave on the inclined rotation axis. Journal of the Korean Earth Science Society, 28, 936-946.

Cheong, H.B. and Park J.R., 2008, On the interpolativeness of discrete Legendre functions. Proceedings of the 2008 International Conference on Scientific Computing, Las Vegas, CSREA press, 252-258.

Cheong, H.B., Park J.R., and Kang H.G., 2012, Fourierseries representation and projection of spherical harmonic functions. Journal of Geodesy, 86, 975-990.

Dilts G.A., 1985, Computation of Spherical harmonic Expansion Coefficients via FFT's. Journal of Computational Physics, 57, 439-453.
Enomoto, T., Fuchigami H., and Shingu S., 2004, Accurate and robust Legendre transforms at large truncation wavenumbers with the Fourier method. Proceedings of the 2004 Workshop on the Solution of Partial Differential Equations on the Sphere, Yokohama, Japan, 17-19.

Hofsommer, D.J. and Potters M.L., 1960, Table of Fourier Coefficients of Associated Legendre Functions. Proceedings of the KNAW, Series A-Mathematical Sciences, Vol 63, Amsterdam, 460-466.

Hopkins, J., 1973, Computation of Normalized Associated Legendre Functions Using Recursive Relations. Journal of Geophysical Research, 78, 476-477.

Jekeli, C., Lee J.K., and Kwon J.H., 2007, On the computation and approximation of ultra-high-degree spherical harmonic series. Journal of Geodesy, 81, 603615.

Moriguchi S.I., Udakawa K.H., and Shin H.M., 1990, Formulas for mathematical functions III. The 5thed., Iwanami Shoten, Tokyo, $310 \mathrm{p}$.

Nehrkorn T., 1990, On the computation of Legendre functions in spectral models. Monthly Weather Review, 118, 2248-2251.

Ricardi L.J. and Burrows M.L., 1972, A recurrence technique for expanding a function in spherical harmonics. IEEE Transactions on Computers 21, 583535.

Risbo T., 1996, Fourier transform summation of Legendre series and D-functions. Journal of Geodesy, 70, 383396.

Rod Blais, J.A., 2008, Discrete spherical harmonic transforms: numerical preconditioning and optimization. Proc. ICCS 2008 Part II (M Bubak et al. Eds.), Springer-Verlag Berlin Heidelberg, LNCS 5102, 683645.

Sardeshmukh P.D. and Hoskins B.J., 1984, Spatial Smoothing on the sphere. Monthly Weather Review, 121, 2524-2529.

Sneeuw, N. and Bun R., 1996, Global spherical harmonic computation by two-dimensional Fourier method. Journal of Geodesy, 70, 224-232.

Swarztrauber, P.N., 1993, The vector harmonic transform method for solving partial differential equations in spherical geometry. Monthly Weather Review, 121, 3415-3437.

Wittwer, T., Klees R., and Seitz K., 2008, Ultra-high degree spherical harmonic analysis and synthesis using extended-range arithmetic. Journal of Geodesy, 82, 223229.

Manuscript received: July 10, 2013 Revised manuscript received: August 13, 2013 Manuscript accepted: August 23, 2013 